\section{Tuberkulosetherapie bei Kindern hocheffektiv}

Gafar F et al. Nationwide analysis of treatment outcomes in children and adolescents routinely treated for tuberculosis in The Netherlands. Eur Respir J 2019; doi:10.1183/13993003.014022019

Mit $>1$ Million erkrankten Kindern im Jahr 2017 gehört die Tuberkulose zu den bedeutendsten globalen Gesundheitsproblemen. Unter den Top Ten in der pädiatrischen Todesursachenstatistik gilt die Tuberkulose als „silent killer“. Nicht nur in einkommensschwachen Ländern kann das Management optimiert werden: Die niederländische, retrospektive Studie zeigt zwar hohe Ansprechraten auf eine Therapie, belegt aber gleichzeitig besondere Risikokonstellationen.

2013 veröffentlichte die WHO einen Leitfaden mit 10 Schlüsselaktionen, die wesentliche Fortschritte in der Bekämpfung der Tuberkulose bei Kindern (TB) brachten. Die Neuauflage von 2018 bezeichnet die Erkrankung als Problem hoher Priorität und schloss Lücken hinsichtlich Prävention und Kontrolle. Auch in Ländern mit einer geringen Inzidenz stellt die TB durch das Fehlen kinderfreundlicher Medikamente und Probleme mit latenten Infektionen (LTBI) eine Herausforderung dar. Die Arbeitsgruppe um Tan evaluierte deshalb die Behandlungsergebnisse und assoziierte Faktoren von 1993-2018 in den Niederlanden behandelten Kindern. Die Daten stammten aus dem Netherlands Tuberculosis Registry NTR. Von insgesamt 3396 Patienten lagen vollständige Daten vor, darunter 51,9\% Kinder ( $<15$ Jahre) und $48,1 \%$ Jugendliche (15-18 Jahre). $57,7 \%$ der Erkrankten waren Jungen und $42,8 \%$ im Ausland geboren. In 42,8\% der Fälle bestand eine Lungentuberkulose. 56,6\% waren in der Kultur positiv ( $94,6 \%$ M. tuberculosis). Die Therapie erfolgte nach dem Standard. Schwere Verläufe mit einem zentralnervösen Befall und Miliartuberkulosen kamen bei 2,9\% vor. Davon waren $44 \%$ nicht BCG-geimpft. Nicht vakzinierte schwere TB betrafen in mehr als der Häfte <5-Jährige.
Von 3253 Patienten lagen die Ansprechraten vor. 95,9\% der Kinder und 92,8\% der Jugendlichen wurden erfolgreich behandelt. Die Ausfallraten (lost to followup LTFU) betrugen 3,4\% und 6,5\%. Ein Behandlungsversagen kam nicht vor. $0,7 \%$ der Population starben. Die multivariate Analyse ergab signifikante Mortalitätsrisiken:

- Alter 2-4 Jahre,

- ZNS-Befall,

- Miliartuberkulose,

- HIV-Koinfektion

- Rezidivbehandlungen und

- medikamentenassoziierte Leberschädigung.

Mehrere Faktoren waren mit einer gesteigerten Wahrscheinlichkeit für LTFU assoziiert. Risikogruppen waren Jugendliche, illegale Immigranten, urbanes Wohnen, unbekannte Ansteckungsquelle, Medikamentenresistenz und -nebenwirkungen sowie Therapieunterbrechungen $>14$ Tage. In einer Subanalyse hatten Jungen, die im Ausland geboren waren, ein gesteigertes LTFU-Risiko.

FAZIT

Die Studie belegte sehr gute Behandlungsergebnisse und identifizierte Risikogruppen für die Mortalität sowie einen Ausfall aus der Studie. Die Untersuchung ergab aber auch protektive Faktoren: Ein „active case finding" und therapiebegleitende Pflegekräfte wirkten sich positiv auf die Therapieeffektivität und die Ausfallraten aus. LTFU sei möglicherweise auch durch die stärkere Nutzung digitaler Medien bei den hochaffinen Jugendlichen zu senken. Besonders wichtig ist auch eine Steigerung der Impfquote, so die Autoren.

Dr. med. Susanne Krome, Melle 